TITLE:

\title{
A method for stiffness tuning of machine tool supports considering contact stiffness
}

\section{AUTHOR(S):}

Kono, Daisuke; Nishio, Syuya; Yamaji, Iwao; Matsubara, Atsushi

\section{CITATION:}

Kono, Daisuke ... [et al]. A method for stiffness tuning of machine tool supports considering contact stiffness. International Journal of Machine Tools and Manufacture 2015, 90: 50-59

\section{ISSUE DATE:}

2015-03

URL:

http://hdl.handle.net/2433/193676

\section{RIGHT:}

(c) 2015 Elsevier Ltd.; この論文は出版社版でありません。引用の際には 出版社版をご確認ご利用ください。; This is not the published version. Please cite only the published version. 


\title{
A method for stiffness tuning of machine tool supports considering contact stiffness
}

\author{
Daisuke Kono ${ }^{1}$, Syuya Nishio ${ }^{1}$, Iwao Yamaji ${ }^{1}$ and Atsushi Matsubara ${ }^{1}$ \\ Corresponding author: Daisuke Kono \\ ${ }^{1}$ Department of Micro Engineering, Graduate School of Engineering, Kyoto University, \\ c1S09, C3,Kyotodaigaku Katsura, Nisikyo-ku, Kyoto 615-8540, Japan \\ Email: kono@prec.kyoto-u.ac.jp \\ Phone: +81-75-383-3677 \\ Fax: +81-75-383-3677
}

\begin{abstract}
A methodology for tuning the stiffness of machine tool supports is described based on a stiffness model using the contact stiffness approach. Using this model, the mathematical relationship between the load of the support and its stiffness is established. The relationship is separated into three regions. When the total stiffness of all supports is maximized, the load must be tuned so that the stiffness-support load relationship is in the critical region, whereby the contact stiffness is slightly larger than the bulk stiffness. Correspondingly, a placement method of supports is proposed that increases their stiffness without anchor bolts. The effectiveness of the proposed method is verified in two experiments. In the first experiment, the natural frequency of a small machine tool prototype is compared for several placements of three supports. The lowest natural frequency of the machine tool under the proposed placement scheme is maximized. In the second experiment, the proposed method is applied to increase the lowest natural frequency of a horizontal milling machine. The lowest natural frequency with a distinct arrangement of three supports is increased by $15 \%-55 \%$, compared to other popular placements of these three supports. The experimental results show that the proposed placement method is effective for enhancing the stiffness of machine tool supports.
\end{abstract}

Keywords: machine tool support, contact stiffness, rocking vibration, stiffness tuning 


\section{Introduction}

Vibration of machine tools must be suppressed to reduce dynamic motion errors in highly productive machining. Ground vibrations transmitted from the floor and drive disturbance vibrations caused by the driving force in feed drives represent the major vibration sources. Invariably, these vibrations excite vibration modes at low frequencies. In particular, rocking vibrations typically represent the lowest two vibration modes of machine tools $[1,2]$. Correspondingly, the dynamic property of the rocking vibration is greatly influenced by the stiffness of supports [2]. Therefore, it is critically important that the stiffness of the supports is properly designed and tuned.

Many studies have previously been reported on the design and the computational evaluation of machine tools. The static and dynamic behaviors of the machine have been evaluated by multi rigid body analysis and finite element analysis (FEA) [3-6]. The optimization of the machine tool design has been studied using these analysis schemes [7-13]. In addition, the fixture design of the workpiece has been also optimized $[14,15]$. However, the design of supports has not been a popular aspect of study in spite of its significant effect on the dynamic performance of machine tools. Rivin discussed the location of supports for reducing the static deformation of the machine [16]. To reduce vibration, Okwudire et al. proposed an optimal location of vibration isolators for an ultraprecision machine tool [17]. Vibration analyses using simplified models were utilized to determine the stiffness of supports for minimizing the vibration $[1,18]$.

However, not many machine tool manufacturers design or tune the stiffness of supports on the basis of dynamic performance evaluation of the machine. One reason is that the stiffness of supports cannot be calculated simply from design parameters, even if FEA is used in a detailed computational model. Proper boundary conditions are required to obtain the stiffness of supports, particularly because the stiffness at contact interfaces has an influence on the stiffness of supports. Although anchor bolts are sometimes used to practically increase the stiffness of supports, their effects have not been clearly explained.

To address this problem, a model based on the contact stiffness of machine tool supports has been developed in one of our previous studies [19]. In this paper, a method for tuning the stiffness of supports is proposed using the developed model. In this approach, a basic idea is first described for tuning the preload and the stiffness of supports. Then, a placement method of supports is proposed, on the basis of increasing their stiffness without the use of anchor bolts. An experimental modal analysis of a small machine tool prototype is then conducted to verify the proposed method. Finally, the proposed method is applied to increase the lowest natural frequency of a horizontal milling machine.

\section{Stiffness tuning of machine tool supports}

\subsection{Stiffness model of machine tool supports based on contact stiffness}

In this study, the stiffness model of machine tool supports, proposed in our previously published study, is used [19]. Figure 1(a) shows the schematic drawing of a screw jack as an example type of a machine tool support. The stiffness of one support is modeled in three-dimensions (3D) as shown in Fig.1(b). The stiffness along each direction, $K$, is obtained from the bulk stiffness and the contact stiffness connected in series, and determined using 


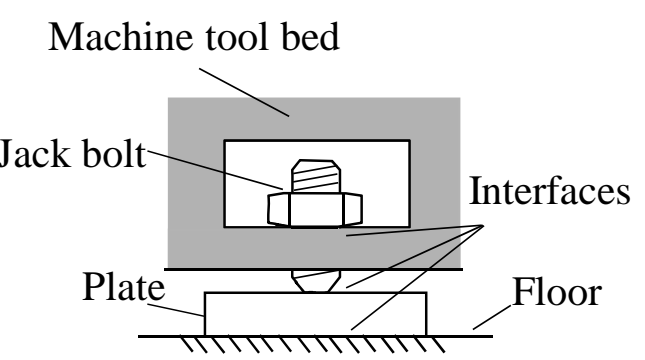

(a) Schematic drawing of screw jack

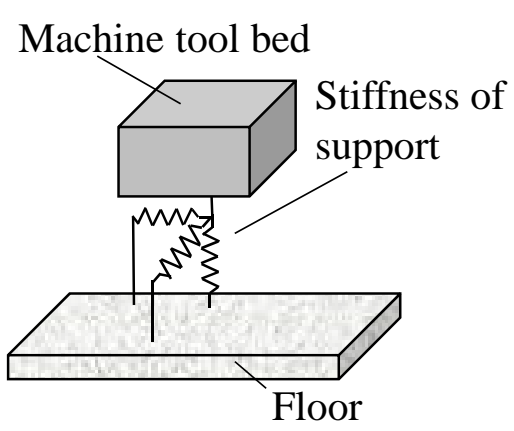

(b) 3D stiffness model of support

Fig. 1 Machine tool support and its model

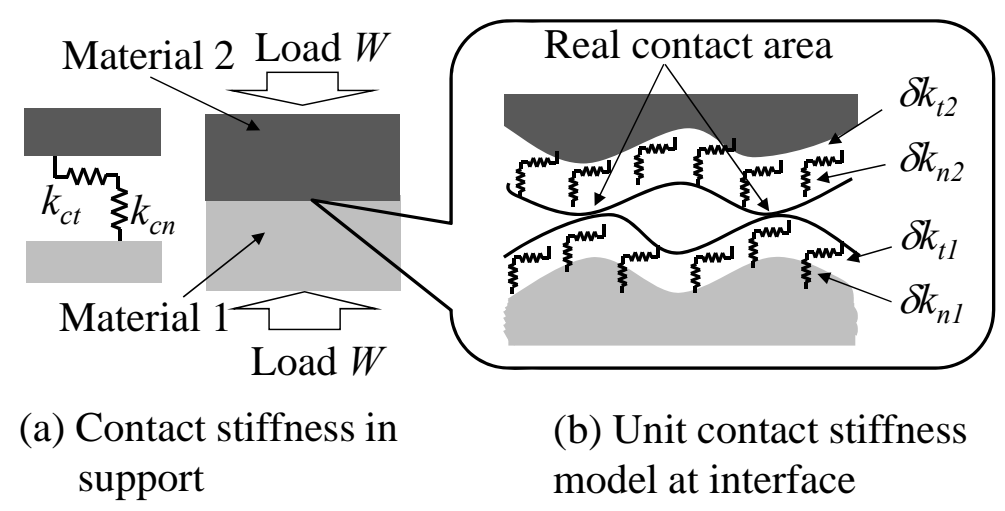

Fig. 2 Model of contact stiffness

$$
\frac{1}{K}=\frac{1}{k_{\text {ball }}}+\frac{1}{k_{\text {call }}}
$$

where $k_{\text {ball }}$ and $k_{\text {call }}$ are the total bulk stiffness and the total contact stiffness for one support, respectively. In an actual case, the support consists of several components and contacted interfaces. Therefore, the above total stiffness is also obtained from their stiffnesses connected in series.

The bulk stiffness can be calculated from the modulus of elasticity and the geometry of the support. The contact stiffness is obtained using the model shown in Fig.2. Figure 2 (a) shows a schematic of an interface at the machine tool support. The preload $W$ acts on the interface; $k_{c n}$ and $k_{c t}$ are the contact stiffnesses in directions normal and tangential to the interface, respectively. The stiffness values $k_{c i}(i=n, t)$ are obtained from a series of coupled springs, spread over the interface using

$$
k_{c i}=\frac{\delta k_{c i 1} \delta k_{c i 2} W}{\left(\delta k_{c i 1}+\delta k_{c i 2}\right) p_{m}}
$$

where the subscript $i$ represents the normal or the tangential direction, $\delta k_{c i l}$ and $\delta k_{c i 2}$ are the contact stiffnesses per unit of real contact area (unit contact stiffness) of materials 1 and 2, respectively, and $p_{m}$ is the lowest value of the two yield pressures associated with materials 1 and 2. 


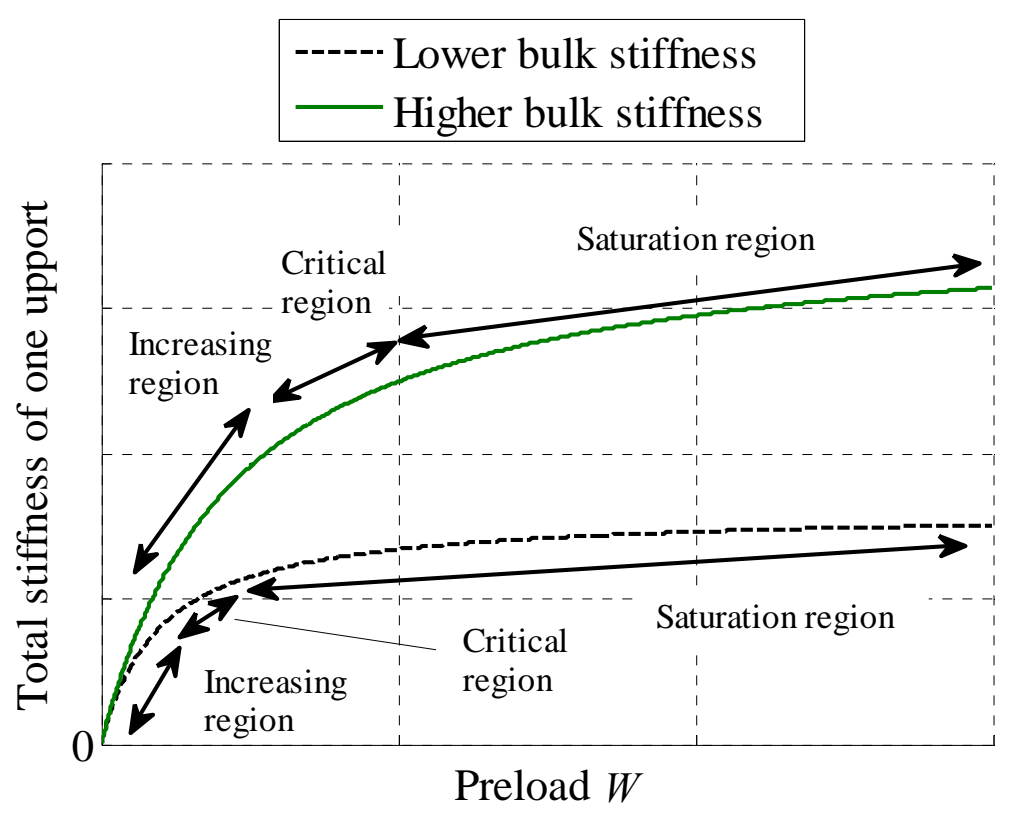

Fig.3 Relationship between preload and stiffness of one support

In the Hertz contact model, the relationship between the preload and the contact stiffness at one roughness asperity on the interface is non-linear and not proportional to the preload. However, in real contact of flat surfaces, the number of contacted asperity increases with the preload. Greenwood et al. have reported that the number of contacted asperity is proportional to the preload and the average size of the contacted spot is constant [20]. Therefore, the real contact area and the contact stiffness increase more sharply than those in the Hertz contact of one asperity. They describes that their discussion can be applied to rough curved surfaces as well as nominally flat surfaces. When the contacted spot is considered as the stiffness spread over the interface, the study by Greenwood et al. supports our contact stiffness model.

\subsection{Stiffness tuning technique}

Generally, if the materials of the support and the floor are not changed, the stiffness of the support can be tuned by either varying the bulk stiffness, the preload, the number of supports or other constitutive parts of the system. A basic idea for selecting any of these approaches is described using the stiffness model presented above.

According to Eqs. (1) and (2), the stiffness of the support depends on the preload. Figure 3 qualitatively shows the relationship between the preload and the stiffness of one support. The relationship is calculated with two different bulk stiffness values using the model described in subsection 2.1. In both cases, the total stiffness is positively correlated with respect to the preload.

The graph representing the total stiffness-preload relationship can be distinguished into the following three regions: the increasing region in which the stiffness increases almost linearly with the preload (because the contact stiffness value is smaller than the bulk value and dominates total stiffness); the saturation region in which the total stiffness is saturated (because the bulk stiffness value dominates the value of total stiffness); and the critical region, which represents the intermediate region between the 


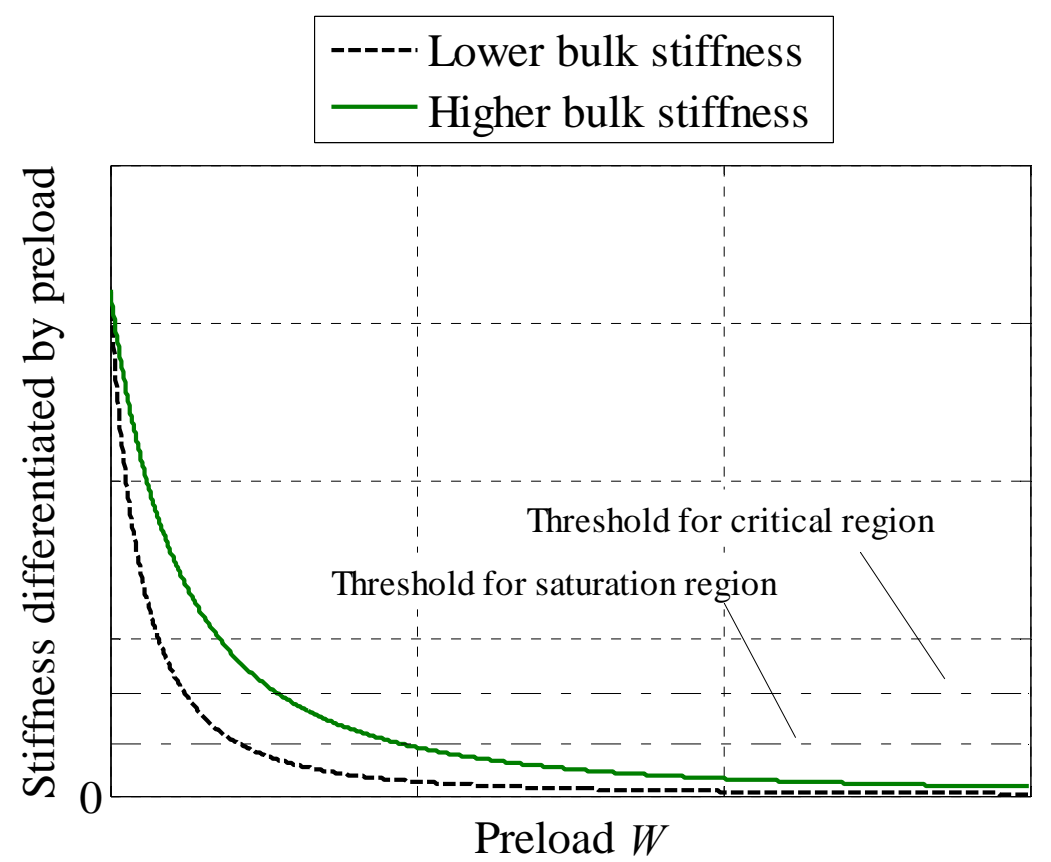

Fig.4 Relationship between preload and stiffness differentiated by preload

increasing and the saturation regions. When the bulk stiffness is increased, the critical region shifts towards larger preload regions and the saturated stiffness increases.

The three regions are used to qualitatively show the efficiency for exchanging the preload to the stiffness. Therefore, the criteria for the regions can be defined using the stiffness differentiated by the preload. Figure 4 shows the relationship between the preload and the stiffness differentiated by the preload. The efficiency is at maximum at $0 \mathrm{~N}$ preload. In this study, the threshold for the boundary between the increasing and the critical regions was determined as $20 \%$ of the maximum value. The threshold for the boundary between the critical and the saturation was determined as $10 \%$. However, these thresholds can be changed because the required efficiency can be different on a case by case.

The stiffness of the support should be tuned according to the total stiffness-preload relationship shown in Fig.3. When the total stiffness of all supports must be increased within the operating range of the increasing region, the preload should be increased by anchor bolts. On the other hand, when operating within in the saturation region, the bulk stiffness should be increased. The number of supports can also be increased within the saturation region because the stiffness does not decrease with decreasing preload.

When the total stiffness of all supports must be decreased, the support can be modified in an opposite manner. Alternatively, the bulk stiffness can be simply decreased because the lower between the bulk and the contact stiffnesses dominates the total stiffness.

When the total stiffness of all supports must be maximized, the support should be modified to maintain the relationship in the critical region because of the following two reasons: the first is the fact that the stiffness can be enhanced in the saturation region by increasing the number of supports, that is, the preload is wasted in this region.; the 
second is that the stiffness can be easily increased within the increasing region by increasing the preloading using anchor bolts.

\subsection{Support placement to increase the stiffness of supports}

As an application of the stiffness tuning idea introduced earlier, the placement of supports is proposed to increase the lowest natural frequency of the machine, by enhancing the total stiffness of all supports without the use of anchor bolts. The preload distribution and the bulk stiffness are changed by changing the position of the support. Then, the following two policies are introduced to determine the support placement;

(1) The preload of the support must be exchanged efficiently to the stiffness using the stiffness- preload relationship.

(2) The distance between the support and the machine gravity center must be long to enhance the rotational stiffness.

The following condition and restriction are also assumed;

- Total preload of all supports is assumed to be constant, given a constant machine mass.

- The object is a machine of small to medium size, which means that the change of gravity center due to moving components does not significantly affect the preload distribution of supports. In addition, the preload is not influenced by disturbances such as the drive disturbance and the process force disturbance.

- The bottom of the bed is almost rectangle.

- The turnover of the machine must be avoided.

Under such policies and assumptions, in general, four corners on the bed are the most possible position for the support.

When four or more supports are used, the support should be placed on the four corners at first. If the preload-stiffness relationship is not in the saturation region, the supports at the four corners are the best because the both policies are satisfied. If the preload-stiffness relationship is in the saturation region, two options are possible to increase the efficiency for exchanging the preload to the stiffness. The first option is to decrease the preload of the support by an additional support. In this case, the stiffness of one support can be slightly decreased. However, the total stiffness of all supports is increased because the number of the support increases. The second option is to increase the bulk stiffness by machine modification. The support position can be also changed to increase the efficiency. However, in terms of the above mentioned policy (2), the rotational stiffness decreases unless the total translational stiffness can be extremely increased by changing the support position.

When three supports are used, all corners cannot be fulfilled. Thus, more options are given for the support placement. The placement procedure is described using an example. Figure 5 shows the footprint of the machine. The origin of the coordinate system is set at the gravity center. To emulate a typical vertical type machine tool, it is assumed that the machine width is larger in the Y direction and that the gravity center of the entire machine is towards its rear side.

Figure 6 outlines the placement procedure with each of the procedural steps being described in the following section.

[Step 1]

The bulk stiffness, $k_{\text {ball }}$, at the proposed support location is calculated, allowing the 


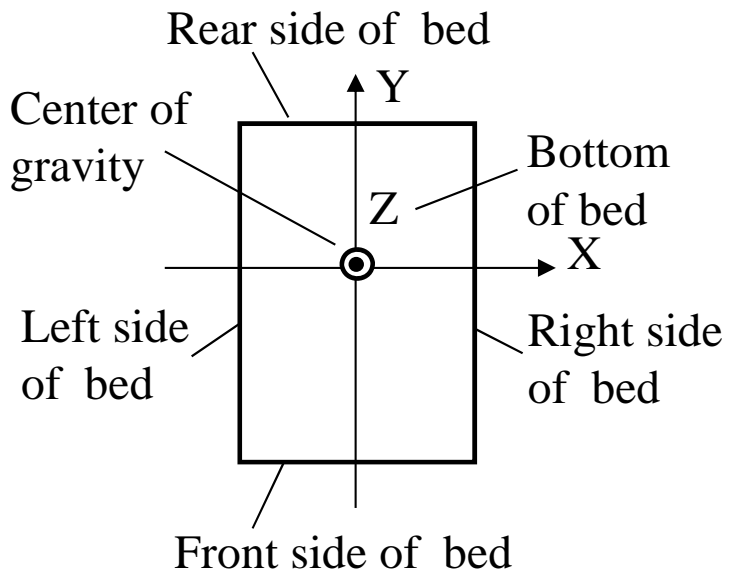

Fig.5 Footprint of machine

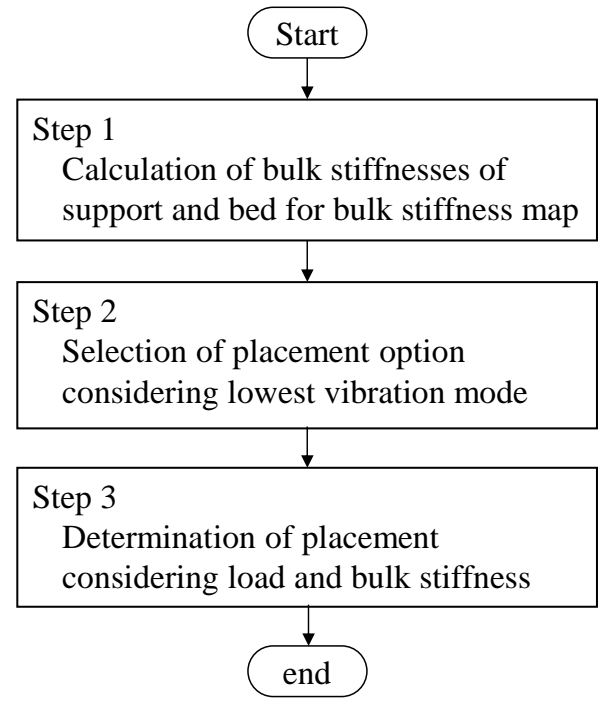

Fig.6 Determination procedure for support location
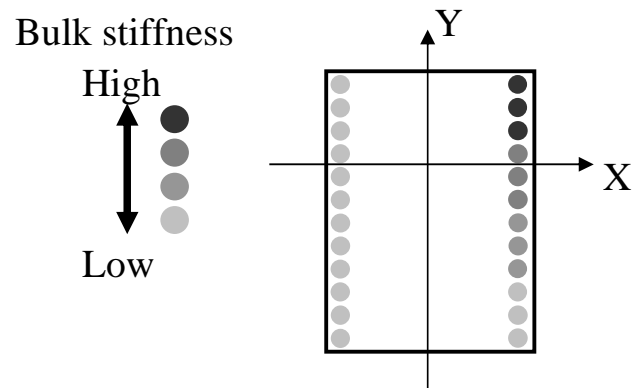

Fig.8 Location candidate for support in Step 2.

Fig.7 Bulk stiffness map in Step 1.

construction of a bulk stiffness map. At this first step, any location on the footprint can be the proposed support location. A method for calculating the bulk stiffness is described in subsection 2.4.

Figure 7 shows an example of the bulk stiffness map. The colored circles represent the proposed location for support placement. The value of the bulk stiffness is codified in a gray scale representation. In this case, the bulk stiffness is nonuniformly distributed for easy understanding.

\section{[Step 2]}

The possible location for support placement is identified to increase the natural frequency of the lowest vibration mode. Figure 8 shows the selected locations. In this case, the rocking vibration, as a result of translations in the $\mathrm{X}$ direction and rotations about the Y direction, is assumed to be the lowest mode. Therefore, the support should be located in the left and right sides of the machine to increase the rotational stiffness about the Y direction. 


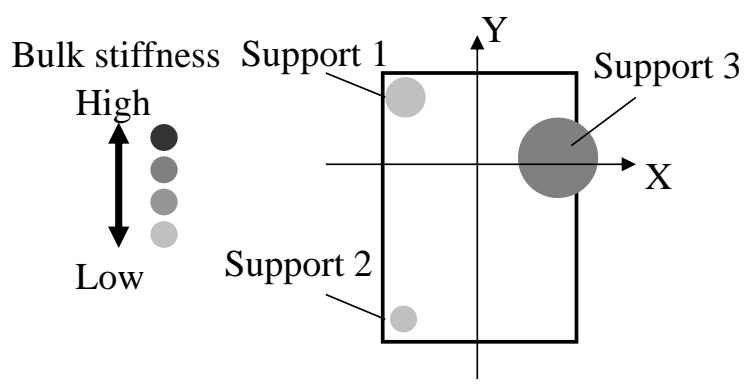

Fig.9 Determined support locaiton. The size of circle represents load of support.

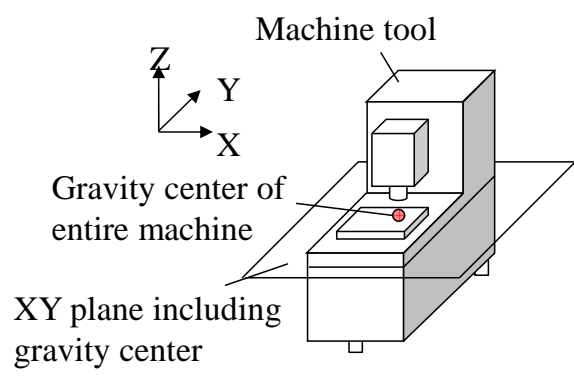

(a) Cross section setting

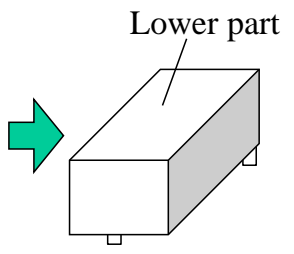

(b) Divide of model

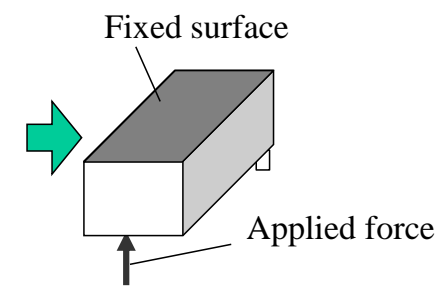

(c) Stiffness calculation

Fig.10 Calculation procedure for bulk stiffness

\section{[Step 3]}

The location is determined considering the preload of the support, the bulk stiffness, and the static stability of the machine. For the proposed location shown in Fig.8, two supports must be located at either the left or the right sides of the machine, and the last support on the opposite side to that occupied by the first two. In this case (Fig. 8), two supports must be located on the left side because its bulk stiffness is lower than that of the right side. Furthermore, the two supports must be located at the front and rear sides to increase the rotational stiffness about the $\mathrm{X}$ direction. Figure 9 shows the determined support locations. The diameter of the circle represents the amount of the preload. The last support is located on the right side to avoid rotational instability of the machine.

\subsection{Bulk stiffness calculation method}

The bulk stiffness of the machine component, from the gravity center to the floor, is a major contributor to the bulk stiffness during rocking vibration. On the basis of this idea, a method for calculating the bulk stiffness is described in this section.

Figure 10 shows the calculation procedure. The bulk stiffness can be calculated from the 3D CAD model of the machine tool using FEA. The cross section is first set in the XY (horizontal) plane, including the gravity center of the entire machine (Fig.10 (a)). Then, the CAD model is divided into two parts, namely the lower and upper parts (Fig.10 (b)). Finally, the bulk stiffness is calculated based on the lower part. The upper surface of this lower part is fixed, and a static force is applied to allow calculation of the 


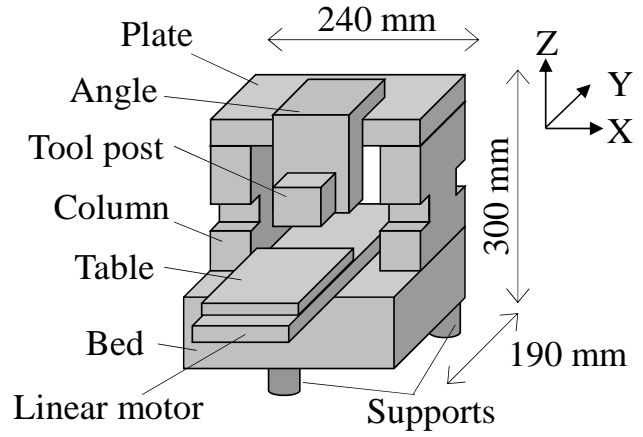

(a) Entire model

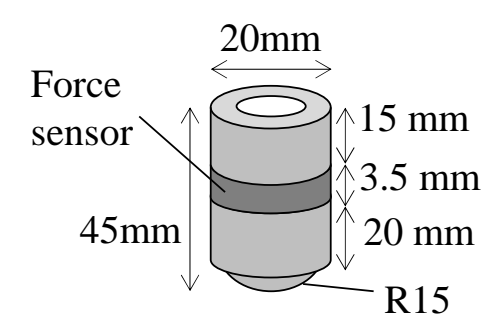

(b) Support

Fig.11 Small model of machine tool used in experiment

Table 1 Specifications of prototype

\begin{tabular}{c|c}
\hline Size & $240 \mathrm{~mm} \times 190 \mathrm{~mm} \times 300 \mathrm{~mm}$ \\
\hline Mass & $53 \mathrm{~kg}$ \\
\hline Drive & Linear motor \\
\hline Guide way & Rolling guide way \\
\hline \multirow{3}{*}{ Material } & $\begin{array}{c}\text { Bed, Column, Table: Cast iron FC250 } \\
\text { Angle, Support: Steel SS400 } \\
\end{array}$ \\
& Plate, Tool post: Steel S50C \\
\hline
\end{tabular}

stiffness (Fig.10 (c)).

In this study, the bulk stiffness must be obtained along the force transmission route from the support and the gravity center of the machine. To obtain this, either of the gravity center or the support is fixed, and a force is loaded on the other. However, when the gravity center is out of the machine body, it is difficult to fix or put a force on it. Thus, a cross section including the gravity center is fixed in this study. The horizontal cross section is used because it must not cross the force transmission route to properly obtain the stiffness.

In most cases, the bulk stiffness in the Z (vertical) direction should be calculated. This is because the stiffness in such a direction is saturated at lower preloads, compared to the stiffness in the $\mathrm{X}$ and $\mathrm{Y}$ (horizontal) directions. This difference arises due to the higher contact stiffness in the vertical compared to the horizontal direction [19].

\section{Verification experiment with a small machine tool prototype}

In the existing machine tool, the location of support is restricted. Therefore, the effect of the proposed method is experimentally verified with a small prototype of the machine tool.

\subsection{Machine tool prototype used in experiments}

Figure 11(a) shows the schematic of the small machine tool prototype used in the experiment. The prototype has a feed drive with a linear motor. Because the bottom face of the bed is flat, the support location is not restricted. The bottom face is finished by grinding. The major specifications of the prototype are listed in Table 1. 


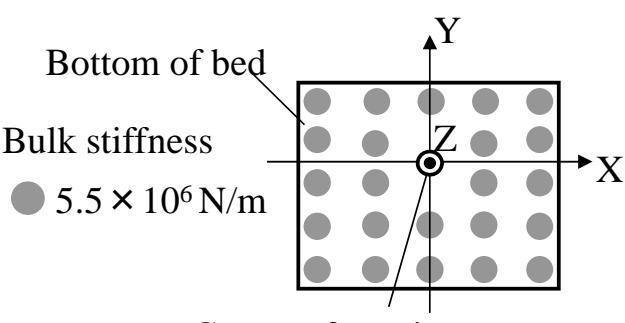

Center of gravity

(a) Step 1

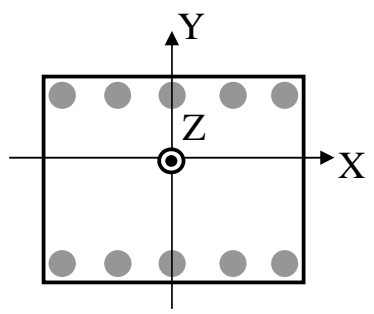

(b) Step 2

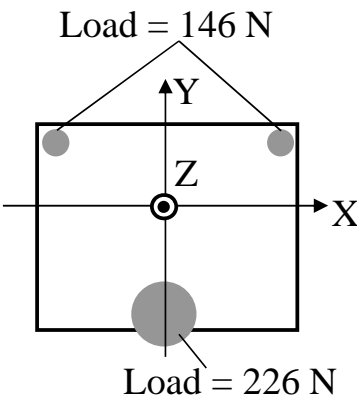

(c) Step 3

Fig.12 Candidate location of supports

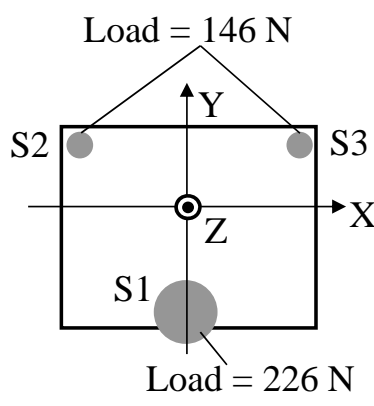

(a) Proposed placement

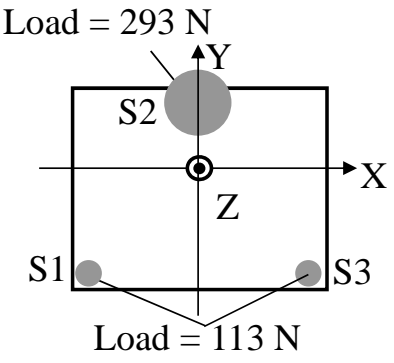

(b) Placement 1

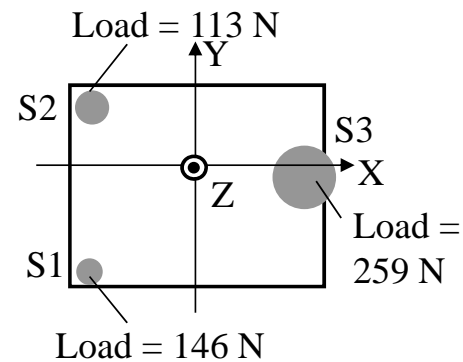

(c) Placement 2

Fig.13 Support placements for comparison

The prototype is mounted on the concrete floor with three (almost cylindrical) supports. Figure 11(b) shows the support schematic. The interface to the bed is finished by grinding and the interface to the floor is rounded by turning. The support contains the force sensor with the sensitivity of $3.8 \mathrm{pC} / \mathrm{N}$ (Kistler) that measures the preload.

3.2 Experiment for increasing the natural frequency of the lowest vibration mode

\subsubsection{Support placement}

The support is located according to the procedure described in subsection 2.3. The bulk stiffness map of the prototype is shown in Fig.12(a). The bulk stiffness in the $\mathrm{Z}$ direction is calculated in this experiment and is uniformly distributed because the bed is a solid block. The possible support locations are selected as shown in Fig.12(b), since their rotational stiffness about the $\mathrm{X}$ direction is enhanced, thereby increasing the natural frequency of the lowest vibration mode. Finally, the support placement is determined as shown in Fig.12(c). The support preload calculated from the support locations and the center of gravity is also shown in the figure. The preload should be uniformly distributed among three supports because the bulk stiffness is uniform. Hence, two supports are located at the side near the center of gravity.

\subsubsection{Experimental method}

The natural frequency of the lowest vibration mode is compared for the three support placements, including the proposed placement scheme, as shown in Fig.13. The 


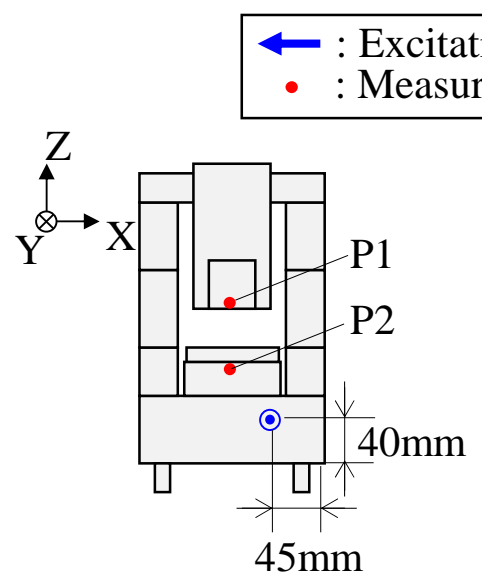

(a) Front view (b) Side view

Fig.14 Excitation and measurement points in experiment

symbols S1, S2 and S3 are used to distinguish the supports. In the scheme under Placement 1 , even if the placement is also suitable for higher rotational stiffness about the $\mathrm{X}$ direction, the preload is concentrated at S2. Therefore, the policy (1) described in subsection 2.3 is not satisfied. In Placement 2, the policy (2) is not satisfied because it maximizes the rotational stiffness about the $\mathrm{Y}$ direction.

The natural frequency is obtained from the modal analysis by impact testing. The prototype is excited in the $\mathrm{Y}$ direction using an impulse hammer (PCB Piezotronics). To obtain a vibration mode shape, a 3D accelerometer (PCB Piezotronics) is used to measure the acceleration at the measurement points, P1-P6, as shown in Fig. 14. The frequency response between the excitation force and acceleration is calculated with a portable fast Fourier transform (FFT)-analyzer (Ono Sokki). The compliance between the excitation force and displacement is obtained by integration. The impulse hammer and the accelerometer sensitivities were found to be $2.3 \mathrm{mV} / \mathrm{N}$ and $50 \mathrm{mV} / \mathrm{m} / \mathrm{s}^{2}$, respectively. The measurement frequency range was set at $400 \mathrm{~Hz}$, and the number of sample points was 2048. The number of averages chosen was five.

\subsubsection{Experimental results}

Figures 15(a) and (b) show the compliances at P1 (tool post) for the X displacement and the Y displacement, respectively. In the magnitude plots of Fig.15(a) and (b), several resonance peaks are observed in the frequency range of 50-80 Hz. These resonances correspond to two rocking vibrations about the $\mathrm{X}$ and the $\mathrm{Y}$ directions. Some results show the presence of two resonance peaks close to each other. This indicates that the prototype vibrates in the diagonal direction along the XY plane in justification of the fact that the rocking vibrations about the $\mathrm{X}$ and the $\mathrm{Y}$ directions are combined.

The natural frequencies of the two rocking vibrations obtained from Fig.15 are summarized in Table 2 . The rocking vibration about the $\mathrm{X}$ direction exhibits the lowest mode under all placement schemes. Table 2 shows that the natural frequency of the lowest mode is highest in the proposed support location. Therefore, the effect of the proposed method for increasing the natural frequency of the lowest vibration mode is verified. 

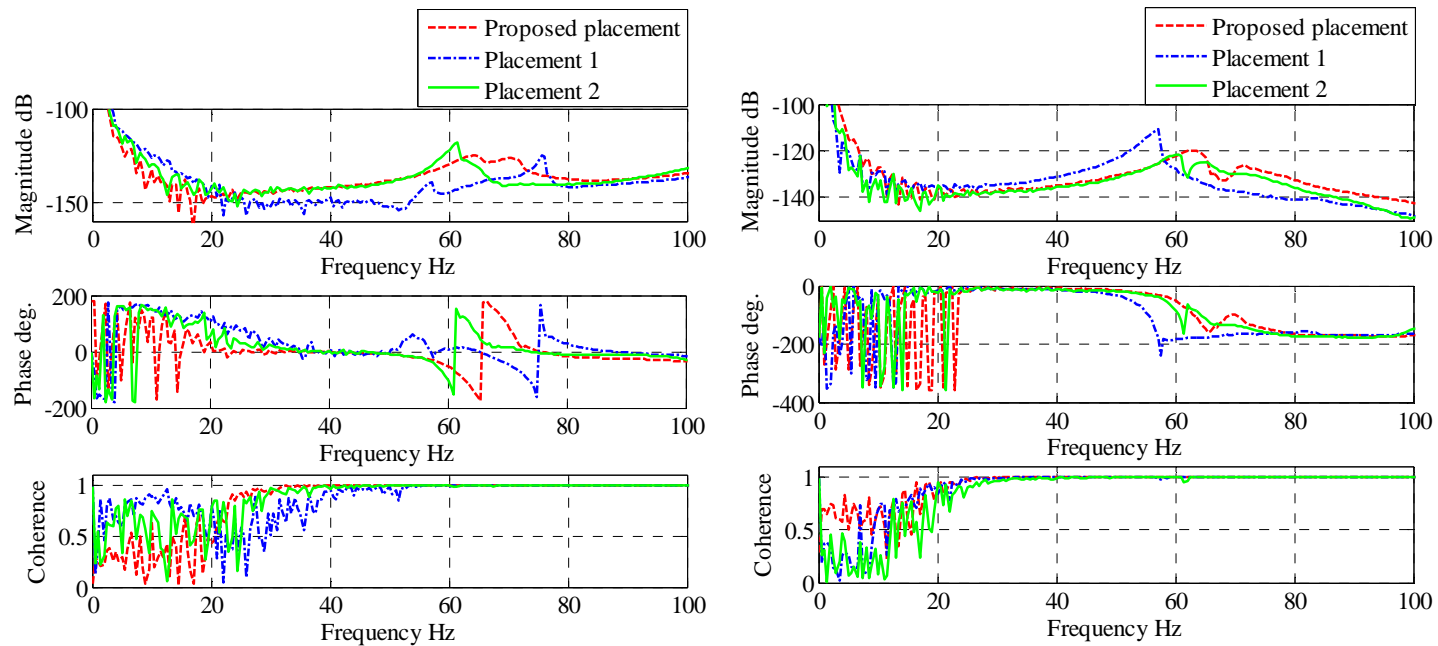

(a) For X displacement

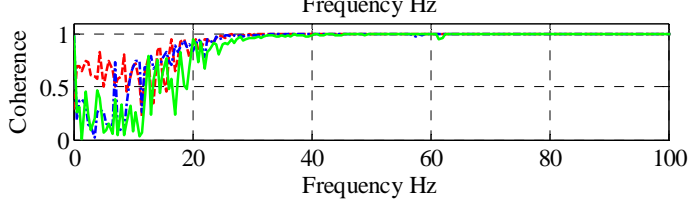

(b) For Y displacement

Fig.15 Compliance measured at measurement point P1 (tool post)

Table 2 Natural frequency of rocking vibration

\begin{tabular}{c|c|c}
\hline \multirow{2}{*}{} & \multicolumn{2}{|c}{ Natural frequency Hz } \\
\cline { 2 - 3 } & $\begin{array}{c}\text { Rocking vibration } \\
\text { around X direction }\end{array}$ & $\begin{array}{c}\text { Rocking vibration } \\
\text { around Y direction }\end{array}$ \\
\hline Proposed location & 63 & 71 \\
\hline Placement 1 & 61 & 65 \\
\hline Placement 2 & 57 & 76 \\
\hline
\end{tabular}

\section{Case study with horizontal milling machine}

In this section, the proposed method is applied to increase the lowest natural frequency of a real machine tool. The natural frequency of the rocking vibration is measured with several placement schemes of supports.

\subsection{Machine tool used in the experiment}

A small knee-type horizontal milling machine is used in this experiment. The photograph of the machine is shown in Fig.16. Major specifications of the machine are listed in Table 3. Leveling blocks were used for the support.

\subsection{Support placement}

\section{[Step 1]}

The placement of supports was decided based on the proposed method. The bulk stiffness is calculated from 3D CAD models of the machine tool and the leveling block using FEA. Figure 17 shows the model of the machine used in the calculation. The major components were represented by solid blocks. The internal part of the column is removed by the shell function to approximately fit the mass of the machine to specifications. The entire model is divided in two parts. The bulk stiffness is calculated 


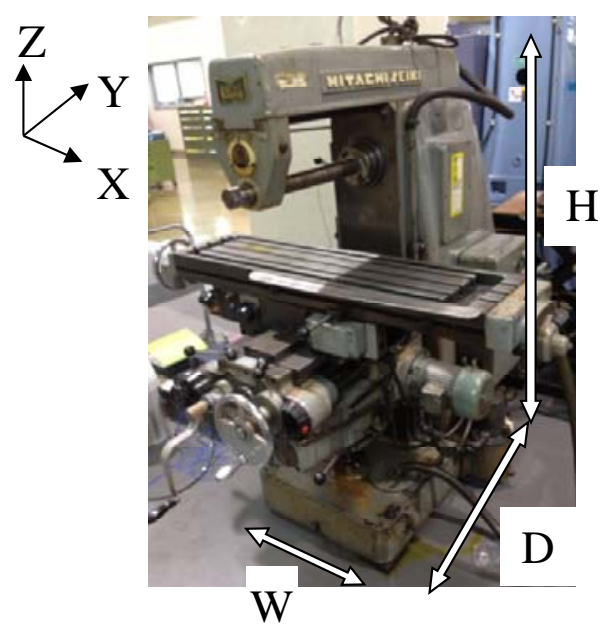

Fig.16 Machine tool used in experiment

Table 3 Specifications of machine tool

\begin{tabular}{|c|c|c|}
\hline & Size & $\mathrm{W}: 500 \mathrm{~mm}, \quad \mathrm{D}: 920 \mathrm{~mm}, \mathrm{H}: 1500 \mathrm{~mm}$ \\
\hline & Mass & $1700 \mathrm{~kg}$ \\
\hline & Stroke & $X: 600 \mathrm{~mm}, \quad Y: 250 \mathrm{~mm}, \quad \mathrm{Z}: 400 \mathrm{~mm}$ \\
\hline & Spindle speed & $60-1800 \mathrm{rpm} / 12$ speeds \\
\hline \multirow{3}{*}{ Support } & Length $\times$ Width $\times$ Hight & $172 \mathrm{~mm} \times 105 \mathrm{~mm} \times 80-85 \mathrm{~mm}$ \\
\hline & Mass & $8 \mathrm{~kg}$ \\
\hline & Allowable load & $15 \mathrm{kN}$ \\
\hline
\end{tabular}

using the lower part, as shown in Fig.17(b).

The material of bodies is set to gray cast iron, which corresponds to a Young's modulus of $110 \mathrm{GPa}$, a density of $7200 \mathrm{~kg} / \mathrm{m}^{3}$, and a Poisson's ratio of 0.28 . The contacted bodies are bonded. The cross section of the column is fixed along all degrees of freedom. The static force of $100 \mathrm{~N}$ is loaded in the positive $\mathrm{Z}$ direction at a measurement position on the bottom of the bed. The displacement at the measurement position is calculated along the $\mathrm{Z}$ direction to allow estimation of the bulk stiffness. The calculation is conducted using ANSYS Workbench, a commercially available FEA software package.

The constructed bulk stiffness map is shown in Fig.18. The colored rectangles represent the candidate location. Similar to prior work, the amount of bulk stiffness is represented in gray scale gradations. The bulk stiffness at the edge of the bed is calculated since the possible support placement position at this edge is selected in Step 2. The bulk stiffness in the rear is larger than the stiffness in the front because the column is fixed.

The preload-stiffness relationship calculated for the experimental machine is shown in Fig.19. The relationship is shown for three bulk stiffnesses which correspond approximately to the stiffnesses at the front side, the right and left sides, and the rear 


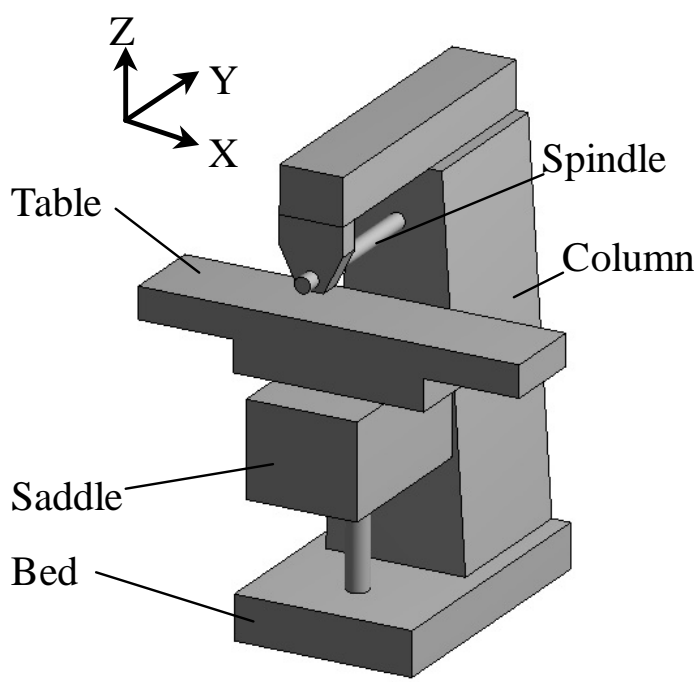

(a) Entire model

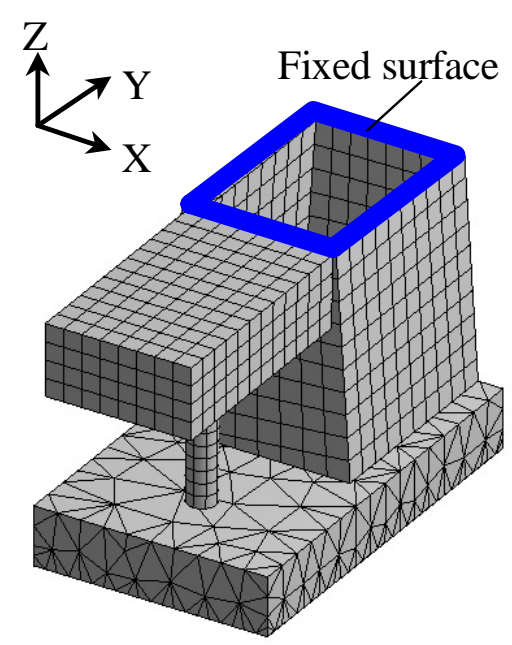

(b) Divided model

Fig.17 3D model of machine tool used in calculation

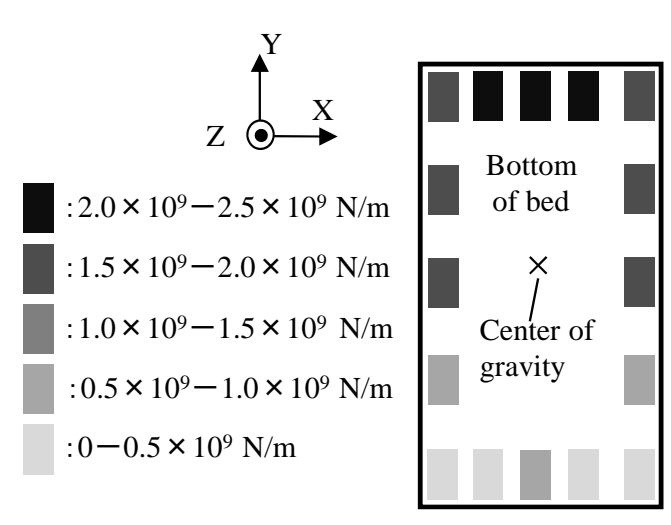

Fig.18 Bulk stiffness map calculated using FEA

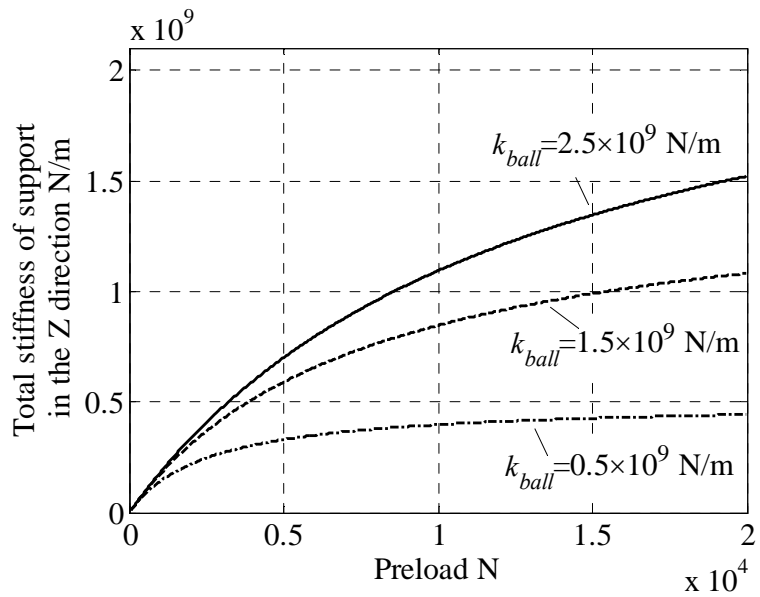

Fig.19 Relationship between preload and stiffness of one support

side of the machine, respectively. The maximum preload of one support is about $8300 \mathrm{~N}$, that is a half of the machine weight. At this preload, the stiffness at the front side starts to saturate. Therefore, the preload on the front side should be limited with care to about $5000 \mathrm{~N}$ or less not to waste the preload.

\section{[Step 2]}

The possible support location is selected to increase the natural frequency of the rocking vibration about the Y direction. Therefore, supports are located on the left and right sides of the machine to increase the rotational stiffness in this direction.

\section{[Step 3]}

Figure 20 illustrates the determined support placement positions. The size of the 


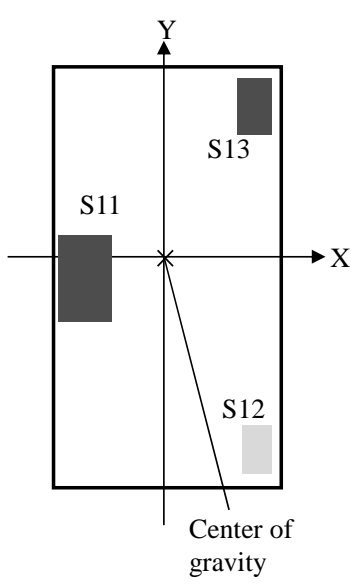

Fig.20 Support placement determined by proposed method
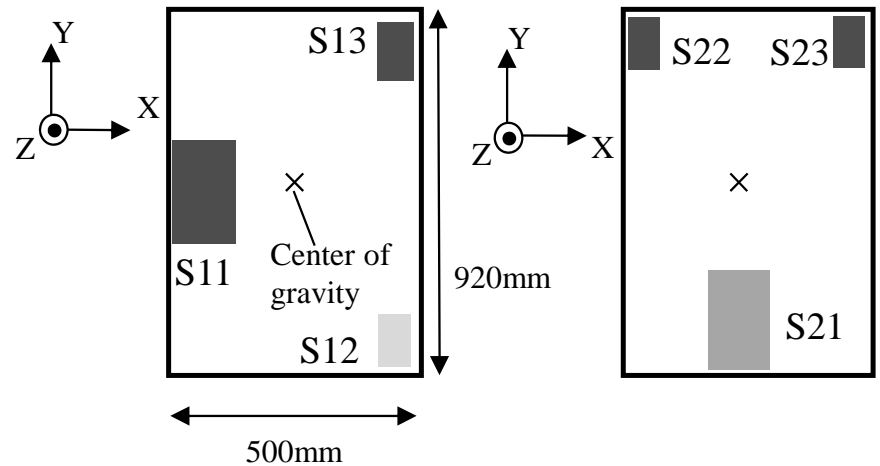

(a) Proposed placement (Placement 1)

(b) Placement 2

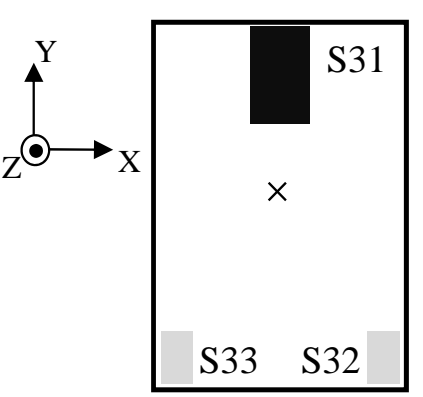

(c) Placement 3

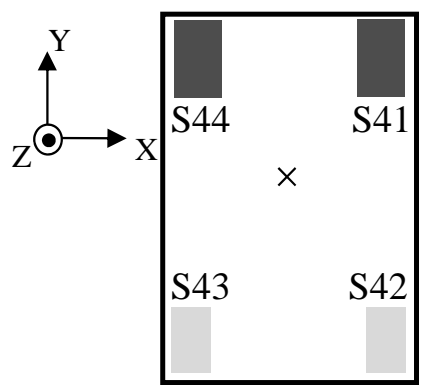

(d) Placement 4

Fig.21 Support placement used in experiment

rectangles qualitatively represents the preload of the support. Symbols S11, S12 and S13 are used to distinguish distinct supports. Because the distribution of the bulk stiffness is symmetric about the Y direction, two supports are located at either the left or the right sides. In this experiment, two supports are located on the right side and the last support on the left side. Two of the supports (S12 and S13) placed at the right side are located at the ends of the front and rear of the machine tool to increase the rotational stiffness about the X direction. The other support (S11) is located slightly shifted along the negative $\mathrm{Y}$ direction to decrease the preload on the support of S12, since the bulk stiffness at $\mathrm{S} 12$ is small.

\subsection{Experimental method}

Figure 21 shows the support placement schemes used in the experiment. Placements 2-4 are the placements for comparison. Placements 2 and 3 are popular placements with three supports. Placement 4 is used to compare the placement schemes with four supports to the best placement scheme with three supports.

The natural frequency is obtained in the modal analysis in a similar way to that adopted by the experiment described in subsection 3.2.2. Briefly, the machine is excited in the $\mathrm{Y}$ direction by an impulse hammer. The measuring apparatus described in section 3.2.2 was also used in this experiment. The measurement and excitation points are shown in Fig.22. Symbols coded under the MP1-16 labels represent the measurement points. The measurement frequency range was set at $400 \mathrm{~Hz}$, and the number of sample 


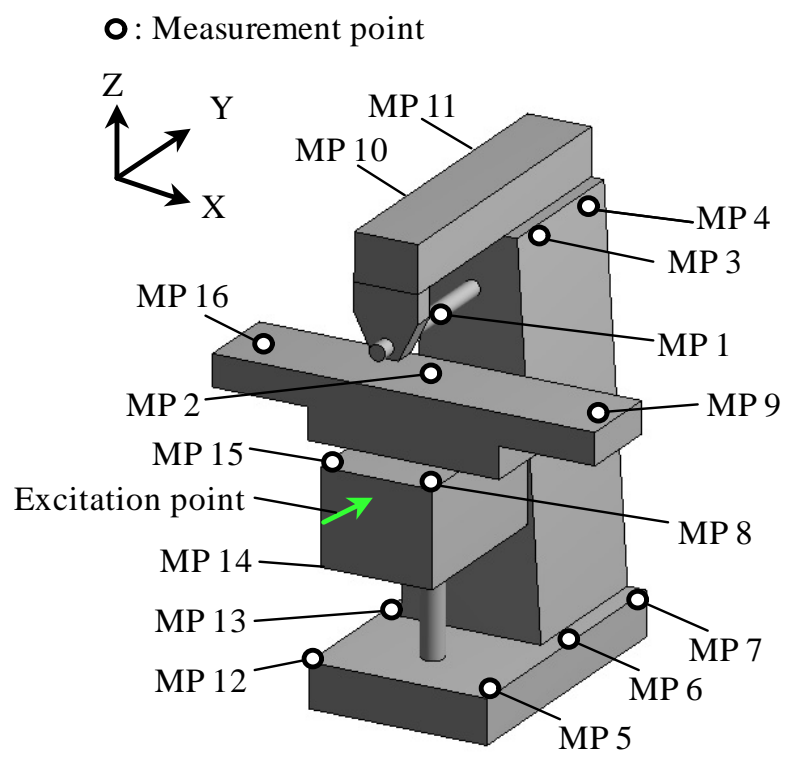

Fig.22 Measurement and excitation points in modal analysis

points was 2048. The number of averaging chosen was five.

\subsection{Experimental result}

The compliance at point MP1 (spindle) is shown in Fig.23. Fig.23(b) shows the magnified view of Fig.23(a). In each result shown in Fig.23(b), two resonance peaks, corresponding to two rocking vibrations are observed. These rocking vibrations occur in the diagonal direction, along the XY plane. In the first mode, the influence of the rocking about the $\mathrm{Y}$ direction was larger. In the second mode, the influence of the rocking about the $\mathrm{X}$ direction was larger.

Table 4 summarizes the natural frequency of the rocking vibrations under the four support placement schemes. The first natural frequency is highest under the proposed placement scheme. With Placements 2 and 3, the first natural frequency is lower than that of the proposed placement scheme, because the policy (2) is not satisfied and the rotational stiffness about the $\mathrm{Y}$ direction is smaller.

The policy (1) is not clearly verified using the proposed placement. This is because the machine is symmetric about the $\mathrm{Y}$ direction and the preload distribution cannot be largely changed like in Chapter 3 . However, the comparison between the second natural frequencies with Placements 2 and 3 shows the effect of the policy (1). In the second vibration mode, the influence of the rocking about the $\mathrm{X}$ direction is larger. The second natural frequency is lower with Placement 2 . Therefore, this results shows that the efficiency is lower with Placement 2 because the preload is concentrated at S21 support. Therefore, this experimental result shows that the natural frequency of rocking vibrations can be enhanced if the proposed method is utilized. 


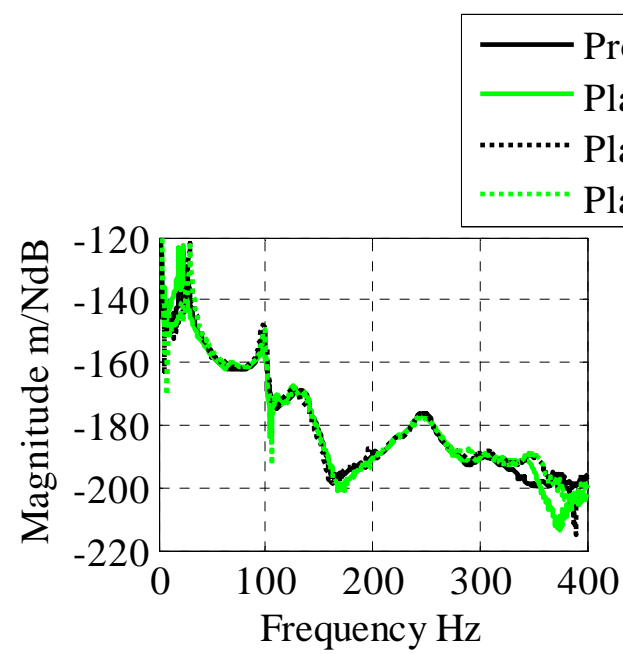

Proposed placement

Placement 2

Placement 3

Placement 4

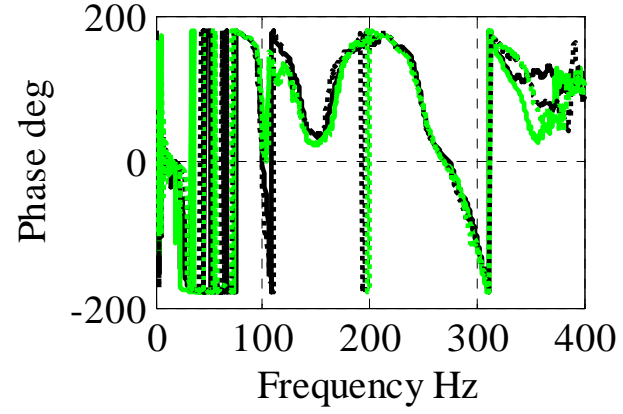

(a) Entire result
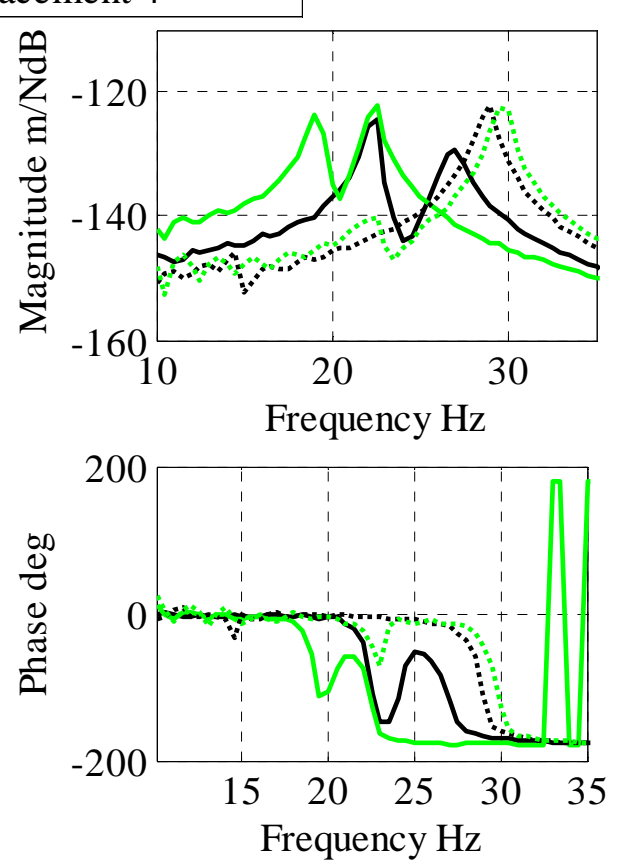

(b) Magnified view

Fig.23 Compliance measured at measurement point MP1 (spindle)

Table 4 Comparison of natural frequencies

\begin{tabular}{c|c|c}
\hline \multirow{2}{*}{ Placement } & \multicolumn{2}{|c}{ Natural frequency Hz } \\
\cline { 2 - 3 } & 1st mode & 2nd mode \\
\hline Proposed placement & 22.5 & 27 \\
\hline Placement 2 & 19 & 22.5 \\
\hline Placement 3 & 14.5 & 29 \\
\hline Placement 4 & 22.5 & 29.5 \\
\hline
\end{tabular}

With Placement 4, the first natural frequency becomes the highest, equal to that of the proposed placement scheme. The second natural frequency under the scheme of Placement 4 is higher compared to that of the proposed placement. Thus, compared to the three-support case, it is noted that a higher natural frequency can be achieved with four supports, if the support preload is uniformly distributed.

\section{Conclusions}

A basic idea for tuning the stiffness of machine tool supports was described based on a stiffness model, using the contact stiffness approach. The relationship between the stiffness and the preload of the support must be considered for tuning the stiffness. When the stiffness should be maximized, the preload should be tuned so that the 
stiffness-preload relationship is in the critical region, in which the contact stiffness is slightly larger than the bulk stiffness. A placement method of supports was proposed that aimed to increase their stiffness without anchor bolts. To verify the proposed placement method, the natural frequency of a small machine tool prototype was measured with several placement schemes of three supports. The lowest natural frequency with the proposed placement scheme was at maximum in such experiments. The proposed method was also applied to increase the lowest natural frequency of a horizontal milling machine. The lowest natural frequency is increased by $15 \%-55 \%$ compared to popular placements schemes of three supports. These experimental results show that the proposed method is effective for enhancing the stiffness of machine tool supports.

\section{Acknowledgement}

This work was supported by KAKENHI (24760104) and Mazak Foundation. 


\section{References}

[1] E.I.Rivin, Vibration isolation of precision equipment, Precision Engineering 17 (1995) 41-56.

[2] K.Yoshida, H.Shimura, H.Yahagi and J.Yoshioka, Effects of mounting elements of surface grinding machines upon their relative receptances between grinding wheel and work table, Journal of Mechanical Working Technology 17 (1988) 377-386.

[3] Y. Altintas, C. Brecher, M. Weck and S. Witt, Virtual Machine Tool, Annals of the CIRP 54 (2005) 115-138.

[4] L. Wang, H. Liu, L. Yang, J. Zhang, W. Zhao and B. Lu, The effect of axis coupling on machine tool dynamics determined by tool deviation, International Journal of Machine Tools \& Manufacture 88 (2015) 71-81.

[5] C.H. Lee, M.Y. Yang, C.W. Oh, T.W. Gim and J.Y. Ha, An integrated prediction model including the cutting process for virtual product development of machine tools, International Journal of Machine Tools \& Manufacture 90 (2015) 29-43.

[6] D.T.Y. Huang and J.J. Lee, On obtaining machine tool stiffness by CAE techniques, International Journal of Machine Tools \& Manufacture 41 (2001) 1149-1163.

[7] D.Huo, K.Cheng and F.Wardle, A holistic integrated dynamic design and modelling approach applied to the development of ultraprecision micro-milling machines, International Journal of Machine Tools \& Manufacture 50 (2010) 335-343.

[8] C.Brecher, P.Utsch, R.Klar and C.Wenzel, Compact design for high precision machine tools, International Journal of Machine Tools \& Manufacture 50 (2010) 328-334.

[9] M.Law, Y.Altintas and A.S.Phani, Rapid evaluation and optimization of machine tools with position-dependent stability, International Journal of Machine Tools \& Manufacture 68 (2013) 81-90.

[10] Z. Yu, K. Nakamoto, T. Ishida and Y. Takeuchi, Interactive design-assistance system of machine tool structure in conceptual and Fundamental design stage, international journal of automation technology 4 (2010) 303-311.

[11] B. Li, J. Hong and Z. Liu, Stiffness design of machine tool structures by a biologically inspired topology optimization method, International Journal of Machine Tools \& Manufacture 84 (2014)33-44.

[12] B.C. Wu, G.S. Young and T.Y. Huang, Application of a two-level optimization process to conceptual structural design of a machine tool, International Journal of Machine Tools \& Manufacture 40 (2000) 783-794.

[13] J.J. Zulaika, F.J. Campa and L.N. Lopez de Lacalle, An integrated process-machine approach for designing productive and lightweight milling machines, International Journal of Machine Tools \& Manufacture 51 (2011) 591-604.

[14] X.J. Wan and Y. Zhang, A novel approach to fixture layout optimization on maximizing dynamic machinability, International Journal of Machine Tools \& Manufacture 70 (2013) 32-44.

[15] E.C. De Meter, Fast support layout optimization, International Journal of Machine Tools \& Manufacture 38 (1998) 1221-1239. 
[16] E.I.Rivin, Stiffness and damping in mechanical design, CRC press, USA, 1999.

[17] C.E.Okwudire and J.Lee, Minimization of the residual vibrations of ultra-precision manufacturing machines via optimal placement of vibration isolators, Precision Engineering 37 (2013) 425-432.

[18] M.Makhult, Machine support design based on vibration calculus, COLLET'S (PUBLISHERS) LTD.,U.K., 1977.

[19] D.Kono, T.Inagaki, A.Matsubara and I.Yamaji, Stiffness model of machine tool supports using contact stiffness, Precision Engineering 37 (2013) 650-657.

[20] J.A. Greenwood and J.B.P. Williamson: Contact of nominally flat surfaces, Proceedings of royal society London A295 (1966) 300-319. 